\title{
Wurtzite materials in alloys of rock salt compounds
}

\author{
Yanbing Han ${ }^{1}$, Samantha L. Millican², Jun Liul ${ }^{3}$, Sage R. Baulers ${ }^{3}$, Sebastian Siol ${ }^{3, b)}$, \\ Stephan Lany ${ }^{3}$, Mowafak Al-Jassim ${ }^{3}$, Charles B. Musgrave ${ }^{2}$, Aaron M. Holder ${ }^{4}$, \\ Andriy Zakutayev ${ }^{3, a)}$ (1) \\ ${ }^{1}$ Materials Science Center, National Renewable Energy Laboratory, Golden, Colorado 80401, USA; and School of Physics and Microelectronics, \\ Zhengzhou University, Zhengzhou 450052, China \\ ${ }^{2}$ Department of Chemical and Biological Engineering, University of Colorado, Boulder, Colorado 80309, USA \\ ${ }^{3}$ Materials Science Center, National Renewable Energy Laboratory, Golden, Colorado 80401, USA \\ ${ }^{4}$ Department of Chemical and Biological Engineering, University of Colorado, Boulder, Colorado 80309, USA; and Materials Science Center, \\ National Renewable Energy Laboratory, Golden, Colorado 80401, USA \\ ${ }^{a}$ Address all correspondence to this author. e-mail: andriy.zakutayev@nrel.gov

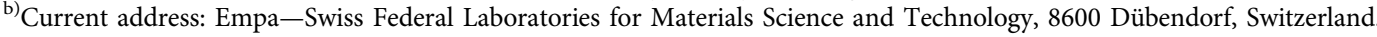 \\ This paper has been selected as an Invited Feature Paper.
}

Received: 15 October 2019; accepted: 13 December 2019

Materials with crystal structures containing tetrahedral motifs are preferable for optoelectronic applications because they often have direct band gaps and low electron effective masses. However, crystal structures of manganese chalcogenides typically contain octahedral motifs, such as in rock salt (RS) MnS and MnSe materials. Here, we experimentally show that $\mathrm{MnS}_{1-x} \mathrm{Se}_{x}$ alloys with tetrahedrally bonded wurtzite (WZ) structure can form between MnSe and MnS parent compounds with octahedral RS structures, at S-rich compositions $(x<0.4)$ and low synthesis temperatures $\left(\sim 300^{\circ} \mathrm{C}\right)$. The calculated mixing enthalpies of $\mathrm{MnS}_{1-x} \mathrm{Se}_{x}$ alloys in $\mathrm{RS}$ and WZ structures cannot explain this experimental observation, so we hypothesize that WZ stabilization may be related to smaller structure density and lower surface energy compared with RS. The resulting WZ $\mathrm{MnS}_{1-x} \mathrm{Se}_{x}$ alloys have 3.0-3.2 eV optical absorption onset and lower electrical conductivity $(<0.0001 \mathrm{~S} / \mathrm{cm})$ than the parent RS compounds. These experimental measurement results are consistent with computationally predicted band gaps and effective masses.

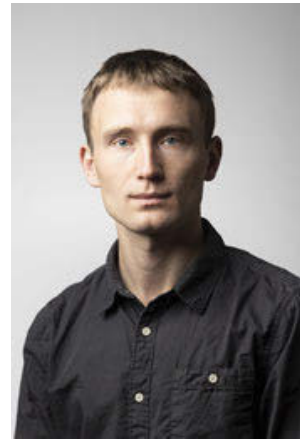

Andriy Zakutayev
Andriy Zakutayev is a scientist at the National Renewable Energy Laboratory (NREL). As an undergraduate student, he studied Electronics at Lviv Polytechnic National University (Ukraine). Dr. Zakutayev received his Ph.D. in Physics in 2010 from Oregon State University (USA), working with Prof. Janet Tate on p-type transparent conductors, including a fellowship with Prof. Andreas Klein at TU Darmstadt (Germany). After 2 years of postdoctoral research with David Ginley and Alex Zunger on transparent conductive oxides at NREL, Andriy started to work on chanlcogenide and nitride photovoltaic absorbers for thin-film solar cells. Currently, Dr. Zakutayev leads a material discovery team at NREL, using high throughput experimental (combinatorial) research methods to design new materials for energy applications. Applied research topics at present include hydrogen production, Li-ion batteries, and power electronic devices. Basic science topics of interest are synthesis of metastable materials using nonequilibrium methods and data science for materials research. Andriy Zakutayev is a recipient of the EMRS Young Scientist Award (2010) and DOE Early Career Award (2019).

\section{Introduction}

Binary metal-chalcogenide semiconductors are $\mathrm{w}$ idely used in optoelectronic devices [1]. Structures containing tetrahedrally coordinated $\left(T_{\mathrm{d}}\right)$ bonding motifs are often preferable for these applications because they have wide direct band gaps and low electron effective masses, and because they are structurally compatible with other chalcogenide materials. For example, CdTe and CdSe used as absorber layers in thin-film solar cells and $\mathrm{ZnS}, \mathrm{ZnSe}$, and CdS used as transport layers in electronics all assume either zinc blende (ZB) or wurtzite (WZ) structures 
$[2,3]$. Manganese chalcogenides are also compelling materials because they are chemically compatible with other binary chalcogenides and have excellent semiconducting properties. For example, $\mathrm{MnO}, \mathrm{MnS}$, and $\mathrm{MnSe}$ are wide band gap materials, MnTe exhibits high p-type conductivity, and $\mathrm{MnCh}$ $(\mathrm{Ch}=\mathrm{S}, \mathrm{Se}, \mathrm{Te})$ are important for instilling magnetism in semiconductors [4]. However, $\mathrm{MnCh}$ ground states contain octahedrally coordinated $\left(O_{\mathrm{h}}\right)$ structural motifs, which are not generally compatible with other commonly used $T_{\mathrm{d}}$ structures such as $\mathrm{WZ}$ and $\mathrm{ZB}: \mathrm{MnO}, \mathrm{MnS}$, and MnSe crystallize in the rock salt (RS) structure, and MnTe crystalizes in the nickeline (NC) structure. Besides, the $\mathrm{MnCh}$ with $\mathrm{O}_{\mathrm{h}}$ structures show indirect band gaps [5], further hindering their optoelectronic applications. Therefore, stabilizing $T_{\mathrm{d}}$ polymorphs of $\mathrm{MnCh}$ may enable their future deployment as active optical materials in optoelectronic devices.

There have been several prior reports about changing $O_{\mathrm{h}}$ structures (like RS and NC) to $T_{\mathrm{d}}$ structures (like ZB and WZ) in $\mathrm{MnCh}$ by heterostructural alloying with $T_{\mathrm{d}} \mathrm{ZnCh}$ leading to novel optoelectronic properties. In the oxides, the insulating RS phase of $\mathrm{MnO}$ was changed to the semiconducting $\mathrm{WZ}$ phase in the $\mathrm{Mn}_{1-x} \mathrm{Zn}_{x} \mathrm{O}$ alloy at $\mathrm{Zn}$ compositions above $x=0.3$ and applied as water splitting photoelectrodes [6]. Similarly, in the selenides, the RS structure of MnSe was converted to the WZ structure in the $\mathrm{Zn}_{1-x} \mathrm{Mn}_{x} \mathrm{Se}$ alloys [7]. In the tellurides, by alloying NC MnTe with trace amount of ZB ZnTe, the ground state switched from the narrow band gap $\mathrm{NC}$ structure to the wide band gap WZ structure [8,9]. Despite the success of alloying for stabilization of $\mathrm{WZ} \mathrm{MnO}, \mathrm{MnSe}$, and $\mathrm{MnTe}$, there is only one report on using alloying to stabilize $\mathrm{WZ} \mathrm{MnS} \mathrm{[4].}$ However, such stabilization of WZ MnS is likely energetically feasible because WZ MnS occurs naturally [10], and has been synthesized via chemical bath deposition [11], heteroepitaxial growth on GaAs [12], and other methods [13].

Studies demonstrating that structures containing $T_{\mathrm{d}}$ motifs can form from alloys containing $\mathrm{MnCh}$ end-member compounds with $O_{\mathrm{h}}$ motifs have been reported. For example, the WZ structure was observed at intermediate compositions in related $\mathrm{MnSe}_{1-x} \mathrm{Te}_{x}$ alloys formed by alloying RS MnSe and NC MnTe [14]. The proposed stabilization mechanism in that case was that the less dense metastable phases ( $T_{\mathrm{d}}$ polymorphs) can form in alloys of more dense end-member compounds $\left(O_{\mathrm{h}}\right.$ structures) due to the lower competition for space in the less dense phases [14]. Depending on the mixing enthalpies and end-member formation energies, this stabilization should also be feasible in iso-structural alloy systems [15]. A computational study was also reported which predicts that $T_{\mathrm{d}}$ phases can form by alloying end-member compounds with the same $O_{\mathrm{h}}$ structures [16]; however, this theory has not been demonstrated experimentally yet. We also note that alloy stabilization of $T_{\mathrm{d}}$ phases by anion substitution (e.g., Se-Te) compared with alloy stabilization by cation substitution (e.g., $\mathrm{Mn}-\mathrm{Zn}$ ) may be more beneficial in optoelectronic devices. This is because in binary compound semiconductors, the conduction band minimum is primarily composed of cation rather than anion states [5], so anion alloying may lead to less perturbation of the low electron effective mass in $T_{\mathrm{d}}$ structures.

Here, we report the stabilization of a structure with $T_{\mathrm{d}}$ bonding motifs in alloys containing two end-member compounds with $O_{\mathrm{h}}$ motifs. Specifically, we find that the WZ structure forms at intermediate compositions in $\mathrm{MnS}_{1-x} \mathrm{Se}_{x}$ alloys, despite end-member MnS and MnSe compounds exhibiting RS structures. Structural and optoelectronic properties of $\mathrm{MnS}_{1-x} \mathrm{Se}_{x}(0 \leq x \leq 1)$ alloy thin films prepared by combinatorial sputtering are reported as a function of chemical compositions and synthesis temperatures. Although films over the entire $\mathrm{MnS}_{1-x} \mathrm{Se}_{x}$ composition range crystalize in the RS structure at higher synthesis temperature $\left(420^{\circ} \mathrm{C}\right)$, at lower temperatures $\left(280-340^{\circ} \mathrm{C}\right)$ the $\mathrm{WZ}$ phase appears at Srich compositions $(x<0.4)$. The optical absorption onset of $\mathrm{WZ} \mathrm{MnS}_{1-x} \mathrm{Se}_{x}$ alloys is $3.0-3.2 \mathrm{eV}$, whereas the conductivity is $<0.0001 \mathrm{~S} / \mathrm{cm}$, indicating its potential for use as a wide band gap semiconductor in various optoelectronic applications. Theoretical mixing enthalpies, electron/hole effective masses, and band gaps are also reported to help better understand the properties of $\mathrm{MnS}_{1-x} \mathrm{Se}_{x}$ alloys, and possible mechanisms of the $\mathrm{WZ}$ phase stabilization are discussed.

\section{Results and discussion}

\section{Structural properties}

Figure 1(a) shows the synthesis phase map of $\mathrm{MnS}_{1-x} \mathrm{Se}_{x}$ alloys as a function of synthesis temperature and chemical composition, based on the X-ray diffraction (XRD) and X-ray fluorescence (XRF) data. As expected, the MnSe and MnS end-member compounds deposited as reference samples exhibit diffraction patterns corresponding to the ground state RS structure at $280-420{ }^{\circ} \mathrm{C}$. However, their crystal structure changes when they form $\mathrm{MnS}_{1-x} \mathrm{Se}_{x}$ alloys at lower temperatures within this range. At $420{ }^{\circ} \mathrm{C}$, the $\mathrm{MnS}_{1-x} \mathrm{Se}_{x}$ alloys have the RS structure across the entire composition range, the same as the end-member compound, and similar to other isostruc-

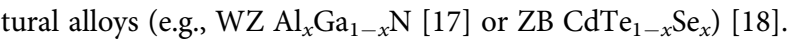
At $340{ }^{\circ} \mathrm{C}$, the $\mathrm{MnS}_{1-x} \mathrm{Se}_{x}$ alloys still exhibit peaks indicative of the RS structure through the whole composition range, but an additional signal from a WZ phase is observed at S-rich compositions $(x<0.3)$. At $280{ }^{\circ} \mathrm{C}$, a similar combination of $\mathrm{RS}+\mathrm{WZ}$ is observed for $0.3<x<0.4$, but for $x<0.3$, the RS signal disappears completely, and all XRD peaks can be attributed to the WZ-phase. Figure 1(b) shows the XRD heat map for $\mathrm{MnS}_{1-x} \mathrm{Se}_{x}$ alloys synthesized at $280{ }^{\circ} \mathrm{C}$, and similar figures for films grown at $420^{\circ} \mathrm{C}$ and $340{ }^{\circ} \mathrm{C}$ that were used to 

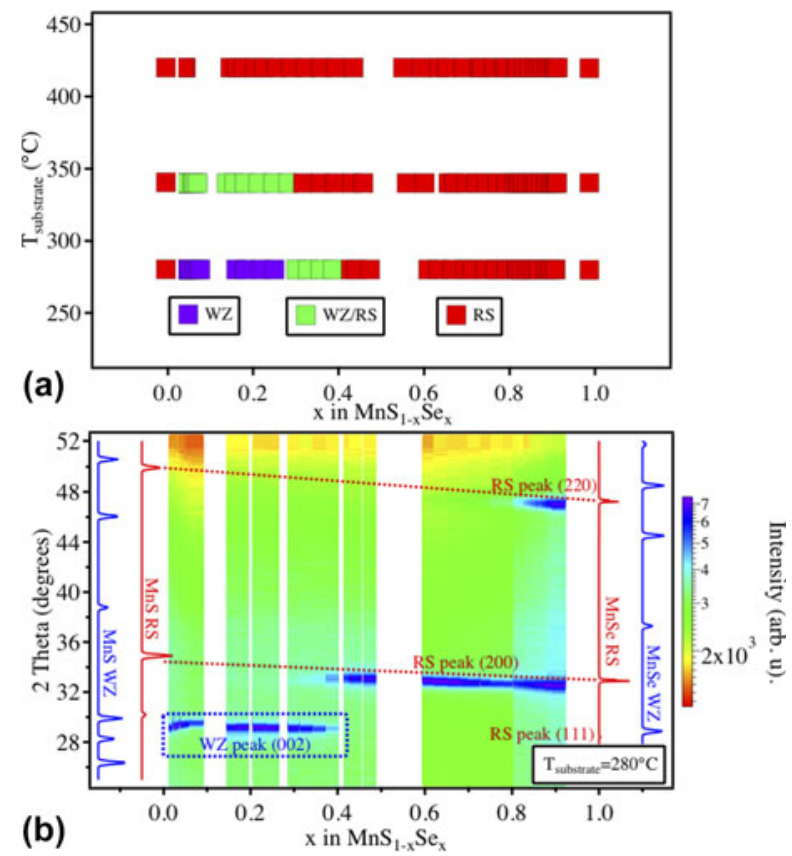

Figure 1: (a) Phase map of $\mathrm{MnS}_{1-x} \mathrm{Se}_{x}$ alloys as a function of chemical composition and synthesis temperature. (b) XRD heat map of $\mathrm{MnS}_{1-x} \mathrm{Se}_{x}$ alloys at $280{ }^{\circ} \mathrm{C}$, showing only WZ peaks appearing at $x<0.3$ composition.

populate points in the phase map can be found in Fig. S1 in the Supplementary material (SM). In the past, similar metastable WZ-MnSe ${ }_{1-x} \mathrm{Te}_{x}$ [14] alloys have been shown to decompose into stable parent compounds close to $400{ }^{\circ} \mathrm{C}$. Overall, these results indicate that the $\mathrm{WZ}$ phase can be obtained under nonequilibrium growth conditions at lower temperatures, where the phase transformation into the RS ground state is kinetically hindered.

To support the WZ phase assignment, a sample with $\mathrm{MnS}_{0.92} \mathrm{Se}_{0.08}$ composition synthesized at $280{ }^{\circ} \mathrm{C}$ was chosen as the representative for more detailed characterization. Figure 2(a) shows the XRD pattern for this sample as a function of $2 \Theta$ angle, with $\chi$ angle integration from $-126^{\circ}$ to $-54^{\circ}$ (detector at $-90^{\circ}$ ), and a fixed $\varphi$ angle. The WZ phase shows strong (002) preferential orientation, with only one prominent peak at $2 \Theta \sim 30^{\circ}$, similar to $\mathrm{WZ} \mathrm{MnS}$ thin films reported in the literature [19]. Comparison of XRD patterns for WZ$\mathrm{MnS}_{0.92} \mathrm{Se}_{0.08}$ with RS-MnS and RS-MnSe is shown in Fig. S2(a) in SM. To further investigate the phase assignment, an XRD pole figure was collected on a different instrument as a function of $\varphi\left(0-360^{\circ}\right)$ and $\chi\left(0-90^{\circ}\right)$ for the measured $2 \Theta$ peak position at $\sim 30^{\circ}$. Figure 2 (b) shows that the peak intensity is strongest at $\chi=90^{\circ}$, where the incident and scattered X-ray plane is normal to the substrate surface, further confirming the $c$-axis preferential orientation of $\mathrm{WZ}$ thin films. Similar full-range XRD measurements were conducted for possible $2 \Theta$ positions of the RS phase, but none of the other

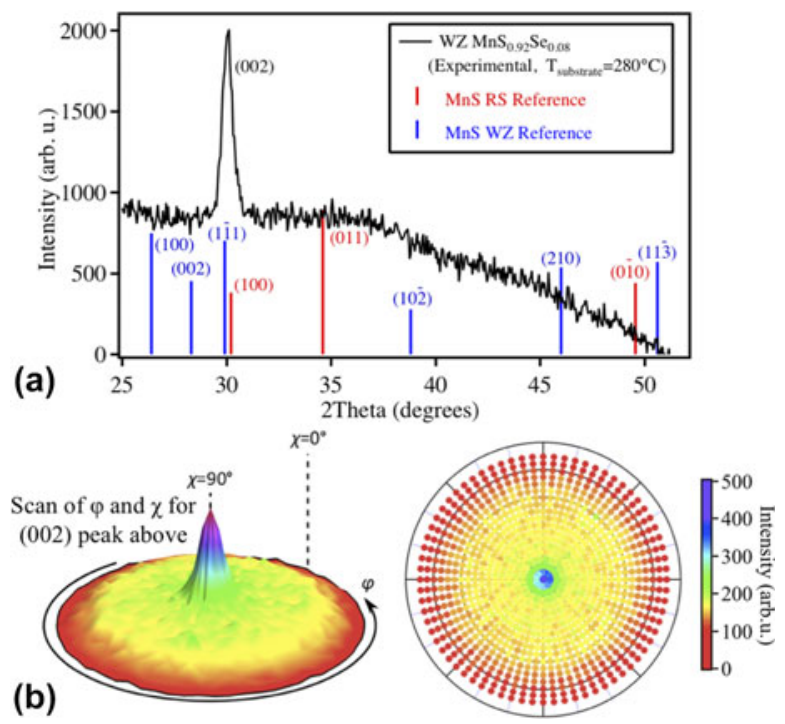

Figure 2: (a) XRD pattern as a function of $2 \Theta$ for $\mathrm{MnS}_{0.92} \mathrm{Se}_{0.08}$ synthesized at $280{ }^{\circ} \mathrm{C}$, showing strong preferential orientation of WZ phase. (b) Full-range XRD scan as a function of $\varphi$ and $\chi$ at $2 \Theta \sim 30^{\circ} \mathrm{WZ}$ peak for $\mathrm{MnS}_{0.92} \mathrm{Se}_{0.08}$ deposited at $280{ }^{\circ} \mathrm{C}$.

peaks were detected, ruling out the presence of the RS phase in $\mathrm{MnS}_{0.92} \mathrm{Se}_{0.08}$ synthesized at $280{ }^{\circ} \mathrm{C}$.

The existence of the $\mathrm{WZ}$ phase in $\mathrm{MnS}_{1-x} \mathrm{Se}_{x}$ alloys is also supported by selected area electron diffraction (SAED) measurements. Figure 3(a) shows the measured SAED pattern for $\mathrm{MnS}_{0.92} \mathrm{Se}_{0.08}$ synthesized at $280{ }^{\circ} \mathrm{C}$ and Fig. 3(b) is the simulated reference pattern based on the [100] zone axis of the $\mathrm{WZ}$ and RS phase. The measured SAED pattern matches well with the simulated $\mathrm{WZ}$ pattern but is different from the RS pattern, and unambiguously confirms the formation of the WZ phase at this composition in $\mathrm{MnS}_{1-x} \mathrm{Se}_{x}$ alloys. However, minor splitting appears for the (0112) peak family, which may be due additional WZ grains with different orientations, or due to minor RS impurity phase. A transmission electron microscopy (TEM) image of this sample point is also provided as Fig. S3 in SM, to show a cross-sectional view of the WZ phase with $\sim 100$-nm grain size that may be possible to increase by annealing. The estimate film thickness from the cross-sectional TEM image is $150 \mathrm{~nm}$, which is similar to $140 \mathrm{~nm}$ determined from the XRF model.

\section{Computational results}

In an attempt to understand the origin of the appearance of the WZ phase between RS end-member compounds, $0 \mathrm{~K}$ mixing enthalpies of $\mathrm{MnS}_{1-x} \mathrm{Se}_{x}$ alloys as function of $x\left(\Delta H_{\mathrm{m}}(x)\right)$ were calculated from first principles, and are shown in Fig. 4(a). Generally, the mixing enthalpy of the RS phase is lower than that of the $\mathrm{WZ}$ phase along the entire composition range, predicting that the alloys should assume the RS phase under equilibrium conditions at $0 \mathrm{~K}$. For the typical synthesis 


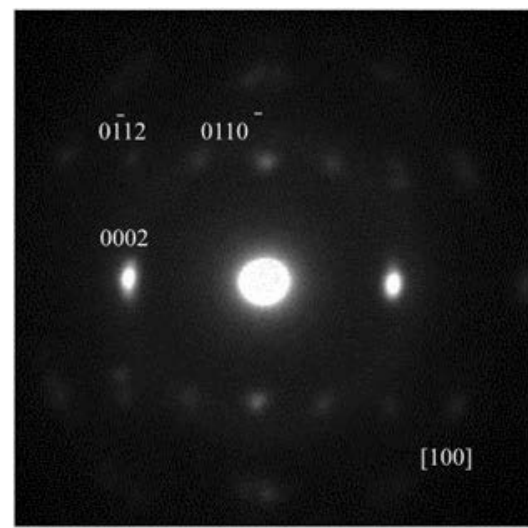

(a)

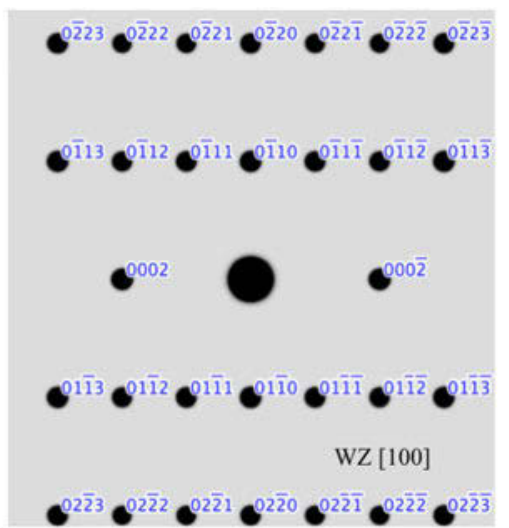

(b)

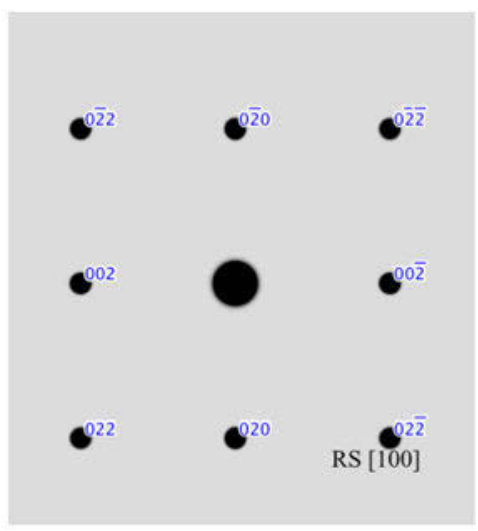

Figure 3: (a) SAED patterns of $\mathrm{MnS}_{0.92} \mathrm{Se}_{0.08}$ grown at $280{ }^{\circ} \mathrm{C}$. (b) Simulated WZ [100] and RS [100] reference pattern from a single crystal.

temperature $\left(280-420^{\circ} \mathrm{C}\right)$, the difference in entropic contribution to the free energy between two solid phases (WZ and RS) is likely to be smaller than the calculated difference in enthalpies of mixing (15-45 meV/at). These computational results are consistent with the measured RS crystal structure of $\mathrm{MnS}_{1-x} \mathrm{Se}_{x}$ alloys synthesized at the higher temperature of $420{ }^{\circ} \mathrm{C}$ [Fig. 1(a)]. However, at $280{ }^{\circ} \mathrm{C}$, the $\mathrm{WZ}$ phase is experimentally observed (Fig. 1) at sulfur-rich compositions. At this lower temperature, formation of the stable RS structure may be kinetically hindered, facilitating formation of metastable WZ structures of the $\mathrm{MnS}_{1-x} \mathrm{Se}_{x}$ alloys. It is particularly surprising that the WZ phase was observed at S-rich compositions [Fig. 1(b)], where the calculated WZ-to-RS polymorph energy difference is the highest [Fig. 4(a)].

Synthesis of the higher energy WZ polymorph in $\mathrm{MnS}_{1-x} \mathrm{Se}_{x}$ alloys by co-sputtering from RS-MnS and RS-MnSe targets (Fig. 1) is quite surprising in light of our theoretical calculations [Fig. 4(a)], but not uncommon in a broader sense. In general, preferential growth of $\mathrm{WZ}$ structure has been observed across different material chemistries (I-VII, II-VI, and II-V) synthesized by a wide range of techniques (solid, liquid, and vapor growths). For example, higher energy WZ polymorphs of I-VII materials ( $\mathrm{LiBr}$ [20] and InCl [21]) have been synthesized by crystallization from amorphous precursors deposited by thermal evaporation on cooled substrates. As another example, preference toward WZ polymorph stabilization at low crystal sizes has been observed in pressure-dependent studies of WZ-to-RS transformations of II-VI (CdSe) nanocrystals prepared by colloidal synthesis techniques [22, 23]. Similar preference of the $\mathrm{WZ}$ over the $\mathrm{ZB}$ polymorph is also known in vapor-liquid-solid (VLS) growth of III-V (GaAs [24] and InAs [25]) nanowires. This broad range of materials and methods suggests a universal mechanism of WZ polymorph preference over other possible structures.

We speculate that the formation of the $\mathrm{WZ}$ phase at S-rich compositions of co-sputtered $\mathrm{MnS}_{1-x} \mathrm{Se}_{x}$ alloys reported here may be related to Ostwald's "step rule" [26]. This rule is based on empirical observations that during the crystallization process metastable structures often form before stable phases. More recently, this rule has been rationalized by measurements of lower surface energies of the metastable polymorphs compared to stable structures [27]. In the case of $\mathrm{MnS}_{1-x} \mathrm{Se}_{x}$ alloys reported here, nucleation of the $T_{\mathrm{d}}$-bonded WZ metastable polymorph may be related to its lower surface energy relative to the $\mathrm{O}_{\mathrm{h}}$-bonded polymorph. Such lower surface energy is likely because of the lower number of broken bonds at the surface of a growing particle, but explicit theoretical calculations would be needed to evaluate this hypothesis. This relation to surface energy is also suggested by the observed strong preferential orientation of the $\mathrm{MnS}_{1-x} \mathrm{Se}_{x}$ alloys along (002) WZ and (111) RS growth directions (Fig. 2), which are structurally related and highly polar. More calculations would be required to rule out potential other hypothesis, such as defect-induced stabilization of the WZ polymorph observed here.

\section{Optoelectronic properties}

Optical properties of $\mathrm{MnS}_{1-x} \mathrm{Se}_{x}$ were also investigated, both experimentally and theoretically. Figure 5 shows the measured absorption heat map of $\mathrm{MnS}_{1-x} \mathrm{Se}_{x}$ as a function of $x$ where an absorption spectrum at each $x$ is displayed as a false color map. The theoretically predicted band gaps of WZ (direct) and RS (indirect) phases are shown as markers for comparison. At $420{ }^{\circ} \mathrm{C}$, the alloys assume the RS phase across the entire composition range [Fig. 5(a)]. Both the predicted and experimental band gaps decrease nearly linearly as $x$ increases, indicating the weak interaction between elemental constituents in $\mathrm{RS} \mathrm{MnS}_{1-x} \mathrm{Se}_{x}$ alloys. This linear change may help to fine-

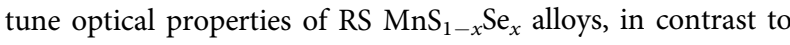
the band gap bowing phenomenon commonly observed in other semiconductors due to the alloying interaction effect 

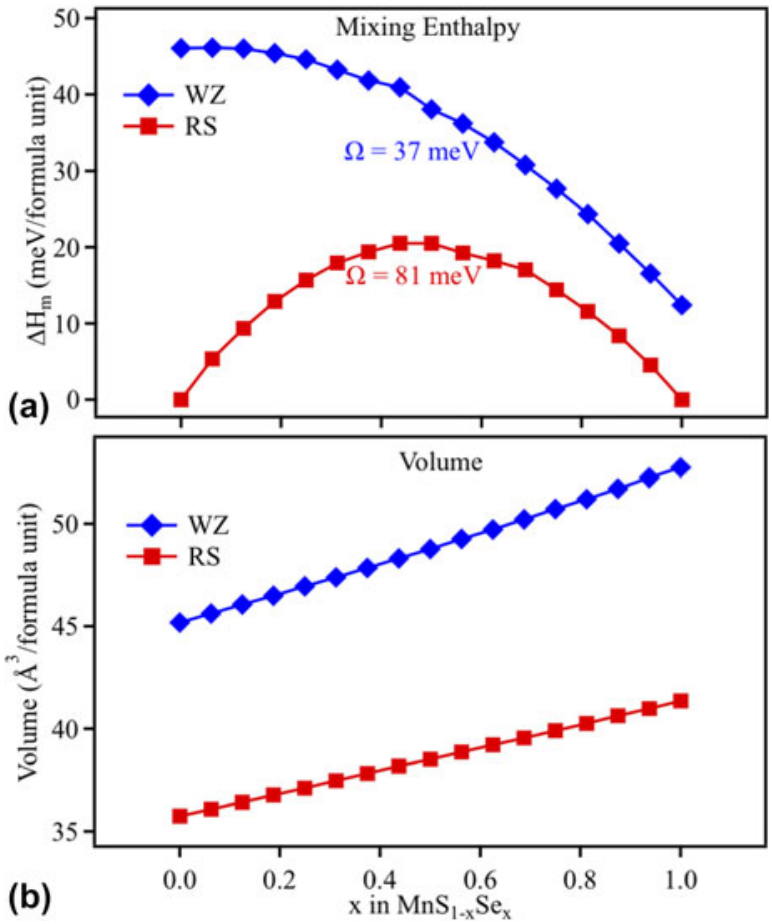

Figure 4: (a) Calculated mixing enthalpy as a function of $x$ in $\mathrm{MnS}_{1-x} \mathrm{Se}_{x}$ (b) Calculated volume per formula unit as a function of $x$ in $\mathrm{MnS}_{1-x} \mathrm{Se}_{x}$

[16]. The measured absorption onset (defined here as an energy at which the absorption coefficient is $10^{5} \mathrm{~cm}^{-1}$ ) is comparable between WZ samples [Fig. 5(b)] and RS samples [Fig. 5(a)] at the same compositions, both in the 3.0-3.2 eV range. The experimental and computational optical absorption onset trends appear cross, as the composition is changed from $\mathrm{MnS}$ to MnSe, but quantification of these differences is difficult, given the present data. Comparison of optical absorption spectra for $\mathrm{WZ}-\mathrm{MnS}_{0.92} \mathrm{Se}_{0.08}$ with RS-MnS and RS-MnSe is shown in Fig. S2(b) in SM. Despite the similar absorption onset, WZ alloys have direct optical band gaps, whereas RS alloys have indirect gaps. The direct band gap of the WZ phase enables its potential application in optoelectronic devices.

Electrical conductivities of the $\mathrm{MnS}_{1-x} \mathrm{Se}_{x}$ alloys synthesized at 420 and $280{ }^{\circ} \mathrm{C}$ are summarized in Fig. 6(a). The conductivities of the $\mathrm{MnS}_{1-x} \mathrm{Se}_{x}$ alloys with a $\mathrm{WZ}$ structure are lower than the measurement limit of the 4-point probe instrument; thus, it is estimated to be below $0.0001 \mathrm{~S} / \mathrm{cm}$. For the $\mathrm{MnS}_{1-x} \mathrm{Se}_{x}$ alloys with a RS structure, the conductivities increase from $0.0001 \mathrm{~S} / \mathrm{cm}$ to $1 \mathrm{~S} / \mathrm{cm}$ with increasing $x$, suggesting that MnSe has shallower defects than MnS. Computationally predicted effective masses of electrons and holes in $\mathrm{MnS}_{1-x} \mathrm{Se}_{x}$ alloys are listed in Fig. 6(b). In $\mathrm{MnS}_{1-x} \mathrm{Se}_{x}$ alloys with a RS structure, the effective masses of electrons and holes both decrease with $x$, which partly explains the increase of conductivities in Fig. 6(a). For the $\mathrm{MnS}_{1-x} \mathrm{Se}_{x}$ alloys with a WZ structure, both effective masses are relatively insensitive
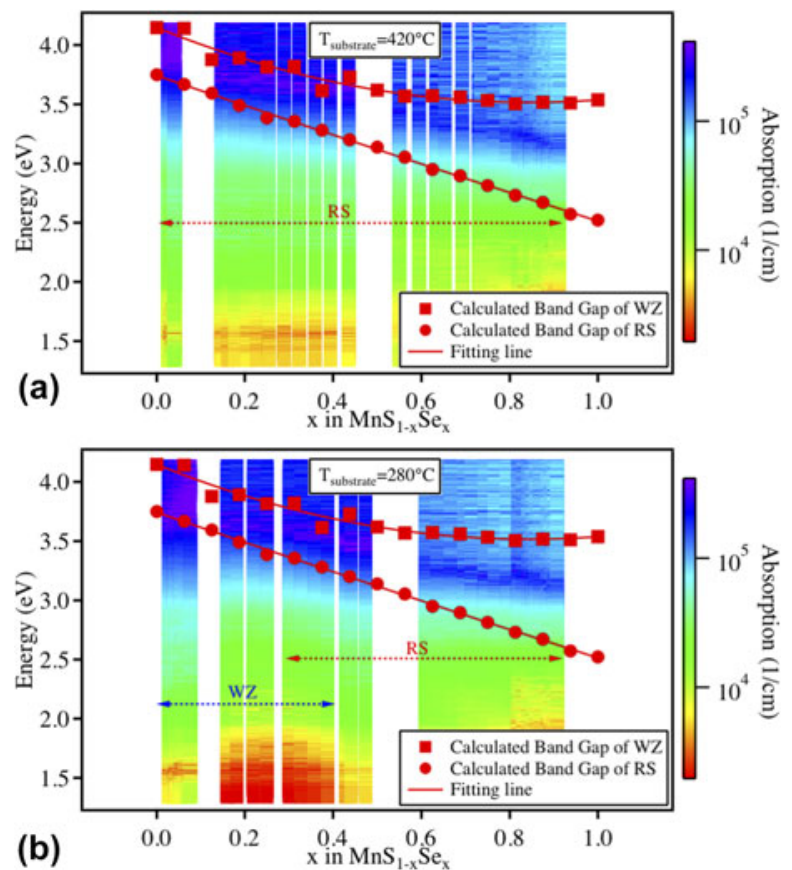

Figure 5: Optical absorption spectra as a function of $x$ in $\mathrm{MnS}_{1-x} \mathrm{Se}_{x}$ alloys synthesized at (a) $420^{\circ} \mathrm{C}$ and (b) $280^{\circ} \mathrm{C}$. The discrete markers are the predicted band gaps of $\mathrm{MnS}_{1-x} \mathrm{Se}_{x}$ alloys in the WZ and RS structures.

to chemical composition $x$, and hole effective mass is quite scattered. This scatter may be related to finite size effect in supercell-based alloy calculations (Fig. S4), which may be amplified in $\mathrm{WZ}$ valence band due to $p-d$ coupling effects [28]. It is also noteworthy that the effective mass of electrons in the WZ structure $\left(0.3 m_{0}\right.$ at $\left.x=0.2\right)$ with a direct band gap is much lower than that of electrons in the RS structure $\left(1.5 m_{0}\right.$ at $x=$ 0.2 ) with an indirect band gap, suggesting potential optoelectronic applications of WZ alloys if they could be doped n-type. Such differences in effective masses between tetrahedrally coordinated (WZ and ZB) and octahedrally coordinated (RS and NC) polymorphs have been reported in other binary chalcogenides $[8,14]$ and in ternary nitrides $[29,30,31]$.

\section{Summary}

The structural and optoelectronic properties of $\mathrm{MnS}_{1-x} \mathrm{Se}_{x}$ alloys $(0 \leq x \leq 1)$ as a function of chemical composition and synthesis temperature are reported. Despite the fact that the end-member compounds $\mathrm{MnS}$ and MnSe assume RS as the experimentally observed and calculated ground state structure, a metastable WZ structure appears at S-rich compositions grown at a lower temperature of $280^{\circ} \mathrm{C}$. This is in contrast to higher temperature growths at $420^{\circ} \mathrm{C}$ where the alloys remain in the RS over the entire composition range. The stabilization of the WZ-phase at lower deposition temperatures is surprising in light of theoretical calculations that predict the mixing enthalpy for WZ structures to be higher than for RS structures 


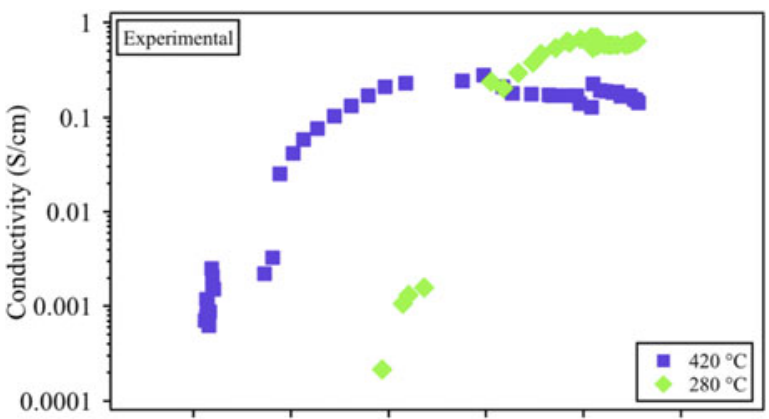

(a)

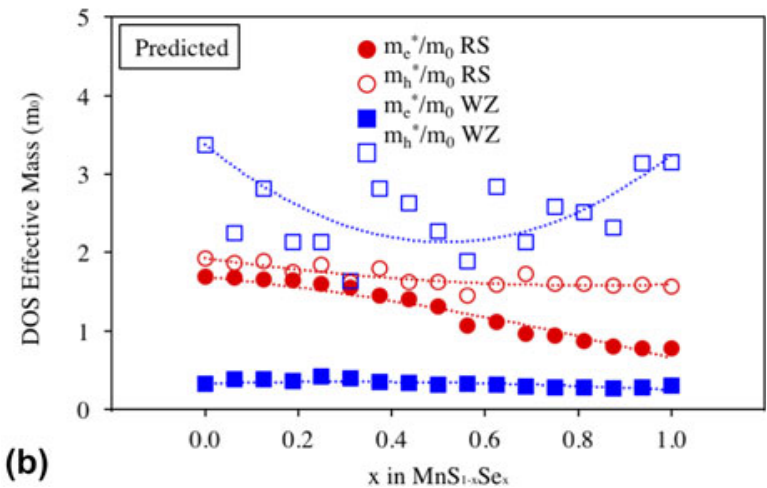

Figure 6: (a) Measured electrical conductivity of $\mathrm{MnS}_{1-x} \mathrm{Se}_{x}$ alloys with the RS structure as a function of $x$. (b) Computationally predicted density of states effective masses $\left(m^{*}\right)$ of electrons and holes in $\mathrm{MnS}_{1-x} \mathrm{Se}_{x}$ alloys with RS and WZ structures.

at all compositions. The stabilization of WZ polymorph may be attributed to lower surface energy of the WZ structure compared with the RS structure, as observed in other materials. In addition, the higher density of RS structure may account for the preference of the formation of WZ phase at S-rich compositions. Computationally predicted band gaps of $\mathrm{MnS}_{1-x} \mathrm{Se}_{x}$ alloys in RS and WZ structures correlate well with the experimentally measured optical absorption onsets. In addition, the RS $\mathrm{MnS}_{1-x} \mathrm{Se}_{x}$ alloys are measured to be more electrically conductive for the Se-rich composition, and calculated electron and hole effective masses are consistent with this observation.

\section{Methods}

\section{Experiments}

$\mathrm{MnS}_{1-x} \mathrm{Se}_{x}(0 \leq x \leq 1)$ alloys were prepared by combinatorial RF sputtering (AJA International, Orion 8). Two sputter targets with a diameter of $50.8 \mathrm{~mm}$, one of $\mathrm{MnSe}$ and one of $\mathrm{MnS}$, were placed opposite to each other in confocal geometry to generate a composition gradient covering the entire composition range in 3-4 depositions. The sputtering power was 8-50 W. For endmember samples, only a single source was used. Fused silica was used as substrates and partially mounted on the sample platen by silver paint. This partial contact induces a thermal gradient across the substrate orthogonal to the composition gradient. The sample platen and substrates were heated by a $\mathrm{SiC}$ heater to achieve the maximum substrate surface temperatures of $420^{\circ} \mathrm{C}$, $340{ }^{\circ} \mathrm{C}$, and $280^{\circ} \mathrm{C}$. The chamber was pumped down to a base pressure of $<1 \times 10^{-6}$ torr before thin films were deposited in high-purity Ar at a working pressure of $1.3 \times 10^{-3}$ torr and an Ar flow rate of $16 \mathrm{sccm}$. The target-substrate distance was $134 \mathrm{~mm}$. More detailed synthesis information can be found in our previous papers $[32,33]$.

After synthesis, the $\mathrm{MnS}_{1-x} \mathrm{Se}_{x}$ thin-film libraries were characterized as a function of position at 44 locations on a rectangular $4 \times 11$ grid. Thin-film properties obtained from these mapping techniques included: composition and thickness measurement by X-ray fluorescence (XRF; Fischerscope XDV$\mathrm{SDD}$ ), crystal structure measured by X-ray diffraction (XRD; Bruker D8) with $\mathrm{Cu} \mathrm{K} \mathrm{K}_{\alpha}$ radiation and a $2 \mathrm{D}$ detector in an approximate Bragg-Brentano geometry, optical transmittance and reflectance measured by a custom UV-Vis instrument using ocean optics spectrometers covering wavelengths of 300$1100 \mathrm{~nm}$, and sheet resistance collected by a custom 4-point probe instrument [34]. The XRF measurements of $\mathrm{MnS}_{1-x} \mathrm{Se}_{x}$ alloy composition were verified by measuring $\mathrm{MnS}$ and $\mathrm{MnSe}$ parent compounds with known composition, and the XRFderived thickness was verified by a cross-sectional TEM measurement. The absorption spectra and electrical conductivities were determined from these measurements based on thickness data obtained from XRF. The large amounts of data that resulted from spatially resolved characterization were analyzed using the COMBIgor software package (https:// www.combigor.com/) [35] and will be made publicly available at HTEM DB (https://htem.nrel.gov/) [36].

The X-ray pole figure of the WZ (002) peak was collected on a Rigaku Smartlab diffractometer using $\mathrm{Cu}-\mathrm{K}_{\alpha}$ radiation and at $2 \Theta=30^{\circ}$. TEM was performed on a FEI Tecnai ST30 at 300 $\mathrm{kV}$. A Pt capping layer was deposited on the surface of the samples to protect the thin-film structure. TEM lamella were prepared by the standard focused ion beam (FIB) lift-out technique and followed by $\mathrm{Ga}$ ion milling to reduce the final thickness to less than $100 \mathrm{~nm}$. Then, a Fischione Nanomill was used to remove preparation damage on the TEM foils using low energy cleaning at $500 \mathrm{eV}$ under vacuum of $10^{-7}$ torr at $-170{ }^{\circ} \mathrm{C}$ for $\pm 10^{\circ}$.

\section{Computations}

Periodic boundary condition density functional theory calculations were performed within the Vienna Ab Initio Simulation Package [37] (VASP) using projector augmented-wave (PAW) pseudopotentials [38]. To obtain accurate polymorph energies of $\mathrm{MnS}$ and $\mathrm{MnSe}$ polymorphs, we used the random phase approximation based on the adiabatic-connection fluctuationdissipation theorem (RPA-ACFDT) [39]. For RPA calculations 
of $\mathrm{MnS}$, the energy cutoff for the plane-wave basis was $360 \mathrm{eV}$ while the energy cutoff for the response function was $240 \mathrm{eV}$ with a 4 atom unit cell. DFT $+U$ with $U=3 \mathrm{eV}$ for Mn- $d$ orbitals was utilized as the input wave function for obtaining the Hartree Fock energy and the ACFDT-RPA correlation energy. The energy difference with respect to the magnetic ground state was determined at the DFT $+U$ level and subtracted from the RPA energy. Polymorph energies for MnSe were calculated in a previous publication in a similar way [14].

For calculations of $\mathrm{MnS}_{x} \mathrm{Se}_{1-x}$ alloy energetics, we used the Perdew-Burke-Ernzerhof (PBE) generalized gradient approximation exchange-correlation functional with a Hubbard correction of $U=3 \mathrm{eV}$ for Mn- $d$ orbitals [40]. Mixing enthalpies were calculated using a 96 atom supercell with random cation configurations generated using the special quasirandom structures method [41]. Calculations were conducted at 17 compositions across the phase space for each crystal structure. Calculations utilized a Gamma centered $2 \times 2 \times 2 k$-point mesh with an energy cutoff of $400 \mathrm{eV}$. Alloy interaction parameters $(\Omega)$ for each polymorph were determined by fitting $\Delta H_{\mathrm{m}}(x)$ using a second-order polynomial.

The theoretical band gaps and DOS effective masses of the alloys were calculated through a linear weighting of the DFTpredicted values to the end-member calculated within the GW approximation [42]. For density of states effective mass calculations, a denser $k$-mesh with $1000 k$-points per reciprocal atom was used, and the results obtained from an energy window corresponding to effective temperatures of 500-3000 $\mathrm{K}$. Correcting the GW self-energy with an attractive on-site potential for the $d$ orbitals has been shown to result in accurate band gaps for transition metal compounds which are generally underestimated by DFT, as described in detail in prior publications [43]. However, the GW method is computationally prohibitive for the large supercells required for the alloys studied here. Therefore, the GW-computed values of the endmembers were used to correct the DFT values for the alloys across the composition space. The computational results of the GW approximation are available from the NREL materials database (http://materials.nrel.gov) [43, 44]. The "effective GW" band gaps and effective masses for alloys of $\mathrm{MnS}_{1-x} \mathrm{Se}_{x}$ are given by a simple linear weighting: $E_{x}^{\mathrm{GW}}=E_{x}^{\mathrm{DFT}}\left(x \frac{E_{x=1}^{\mathrm{GW}}}{E_{x=1}^{\mathrm{DVT}}}+\frac{(1-x) E_{x=0}^{\mathrm{GW}}}{E_{x=0}^{\mathrm{DT} T}}\right)$, as described in previous publications [45]. This approach is reasonable because the band gaps and effective masses are underestimated by DFT, but the changes in the electronic structure across the composition range are generally correct in these materials.

\section{Acknowledgments}

This work was supported by the U.S. Department of Energy under Contract No. DE-AC36-08GO28308 with Alliance for
Sustainable Energy, LLC, the Manager and Operator of the National Renewable Energy Laboratory. Funding provided by Office of Science, Office of Basic Energy Sciences, as part of the Energy Frontier Research Center "Center for Next Generation of Materials Design: Incorporating Metastability.” Y.H. thanks the China Scholarship Council for offering the stipend to perform research at the National Renewable Energy Laboratory. S.L.M. also acknowledges the support from the U.S. Department of Energy's Office of Energy Efficiency and Renewable Energy, Fuel Cells Technologies Office Award No. DE-EE0008088, "Computationally Accelerated Discovery and Experimental Demonstration of High-Performance Materials for Advanced Solar Thermochemical Hydrogen Production.” The computational research was performed using resources sponsored by the Department of Energy's Office of Energy Efficiency and Renewable Energy and located at the National Renewable Energy Laboratory. The authors would like to thank Angela Fioretti, Kevin Talley, and Yun Xu for useful discussions and valuable suggestions. The views expressed in the article do not necessarily represent the views of the DOE or the U.S. government.

\section{Supplementary material}

To view the supplementary material for this article, visit https://doi.org/10.1557/jmr.2019.402.

\section{References}

1. H. Singh, T. Singh, and J. Sharma: Review on optical, structural and electrical properties of ZnTe thin films: Effect of deposition techniques, annealing and doping. ISSS J. Micro Smart Syst. 7, 123 (2018).

2. T.D. Lee and A.U. Ebong: A review of thin film solar cell technologies and challenges. Renewable Sustainable Energy Rev. 70, 1286 (2017).

3. F. Huang, Q. Zhang, B. Xu, J. Hou, Y. Wang, R.C. Massé, S. Peng, J. Liu, and G. Cao: A comparison of $\mathrm{ZnS}$ and $\mathrm{ZnSe}$ passivation layers on CdS/CdSe co-sensitized quantum dot solar cells. J. Mater. Chem. A 4, 14773 (2016).

4. J.K. Furdyna: Diluted magnetic semiconductors. J. Appl. Phys. 64, R29 (1988).

5. S.J. Youn, B.I. Min, and A.J. Freeman: Crossroads electronic structure of MnS, MnSe, and MnTe. Phys. Status Solidi 241, 1411 (2004).

6. H. Peng, P.F. Ndione, D.S. Ginley, A. Zakutayev, and S. Lany: Design of semiconducting tetrahedral $\mathrm{Mn}_{1-x} \mathrm{Zn}_{x} \mathrm{O}$ alloys and their application to solar water splitting. Phys. Rev. X 5, 021016 (2015).

7. J. Xue, Y. Ye, F. Medina, L. Martinez, S.A. Lopez-Rivera, and

W. Giriat: Temperature evolution of the $2.1 \mathrm{eV}$ band in the $\mathrm{Zn}_{1-x} \mathrm{Mn}_{x} \mathrm{Se}$ system for low concentration. J. Lumin. 78, 173 (1998). 
8. Y. Han, A.M. Holder, S. Siol, S. Lany, Q. Zhang, and

A. Zakutayev: Zinc-stabilized manganese telluride with wurtzite crystal structure. J. Phys. Chem. C 122, 18769 (2018).

9. A.Z. Sebastian Siol, Y. Han, J. Mangum, P. Schulz, A.M. Holder, T.R. Klein, M.F.A.M. van Hest, and B. Gorman: Stabilization of wide band-gap p-type wurtzite MnTe thin films on amorphous substrates. J. Mater. Chem. C 6, 6297 (2018).

10. M.P. Kalinowski: Rambergite, a new polymorph of $\mathrm{MnS}$ with hexagonal structure. GFF 118, 53 (1996).

11. C. Gümüş, C. Ulutaş, R. Esen, O.M. Özkendir, and Y. Ufuktepe: Preparation and characterization of crystalline MnS thin films by chemical bath deposition. Thin Solid Films 492, 1 (2005)

12. M. Okajima and T. Tohda: Heteroepitaxial growth of MnS on GaAs substrates. J. Cryst. Growth 117, 810 (1992).

13. J. Beltran-Huarac, J. Palomino, O. Resto, J. Wang, W.M. Jadwisienczak, B.R. Weiner, and G. Morell: Highlycrystalline $\gamma$-MnS nanosaws. RSC Adv. 4, 38103 (2014).

14. S. Siol, A. Holder, J. Steffes, L.T. Schelhas, K.H. Stone,

L. Garten, J.D. Perkins, P.A. Parilla, M.F. Toney, and B.D. Huey: Negative-pressure polymorphs made by heterostructural alloying. Sci. Adv. 4, 1442 (2018).

15. S. Siol: Accessing metastability in heterostructural semiconductor alloys. Phys. Status Solidi 1800858 (2019).

16. S. Lany: Polymorphism, band-structure, band-lineup, and alloy energetics of the group II oxides and sulfides $\mathrm{MgO}, \mathrm{ZnO}, \mathrm{CdO}$, $\mathrm{MgS}, \mathrm{ZnS}$, CdS. In Proceedings of Oxide-based Materials and Devices V, International Society for Optics and Photonics, vol. 8987 (SPIE OPTO, San Francisco, California, 2014); p. 89870K.

17. H. Morko: Handbook of Nitride Semiconductors and Devices (Wiley, Hoboken NJ, USA, 2008).

18. K.W. Böer: Handbook of the Physics of Thin-Film Solar Cells (Berlin, Germany, Springer Science \& Business, 2014).

19. C. Gümüş, C. Ulutaş, and Y. Ufuktepe: Optical and structural properties of manganese sulfide thin films. Opt. Mater. 29, 1183 (2007).

20. Y. Liebold-Ribeiro, D. Fischer, and M. Jansen: Experimental substantiation of the "energy landscape concept" for solids: Synthesis of a new modification of LiBr. Angew. Chem., Int. Ed. 47, 4428 (2008).

21. A. Bach, D. Fischer, and M. Jansen: Metastable phase formation of indium monochloride from an amorphous feedstock. Z. Anorg. Allg. Chem. 639, 465 (2013).

22. S.H. Tolbert and A.P. Alivisatos: Size dependence of a first order solid-solid phase transition: The wurtzite to rock salt transformation in CdSe nanocrystals. Science 265, 373 (1994).

23. C-C. Chen, A.B. Herhold, C.S. Johnson, and A.P. Alivisatos: Size dependence of structural metastability in semiconductor nanocrystals. Science 276, 398 (1997).
24. F. Glas, J-C. Harmand, and G. Patriarche: Why does wurtzite form in nanowires of III-V zinc blende semiconductors? Phys. Rev. Lett. 99, 146101 (2007).

25. M. Koguchi, H. Kakibayashi, M. Yazawa, K. Hiruma, and T. Katsuyama: Crystal structure change of GaAs and InAs whiskers from zinc-blende to wurtzite type. Jpn. J. Appl. Phys. 31 2061 (1992)

26. W. Ostwald: Studien über die Bildung und Umwandlung fester Körper. Z. Phys. Chem. 22, 289 (1897).

27. A. Navrotsky: Energetic clues to pathways to biomineralization: Precursors, clusters, and nanoparticles. Proc. Natl. Acad. Sci. 101, 12096 (2004).

28. S.H. Wei and A. Zunger: Role of metal $d$ states in II-VI semiconductors. Phys. Rev. B 37, 8958 (1988).

29. S.R. Bauers, A. Holder, W. Sun, C.L. Melamed, R. WoodsRobinson, J. Mangum, J. Perkins, W. Tumas, B. Gorman, A. Tamboli, G. Ceder, S. Lany, and A. Zakutayev: Ternary nitride semiconductors in the rocksalt crystal structure. Proc. Natl. Acad. Sci. U. S. A. 116, 14829 (2019).

30. E. Arca, J.D. Perkins, S. Lany, A. Mis, B-R. Chen, P. Dippo, J.L. Partridge, W. Sun, A. Holder, A.C. Tamboli, M.F. Toney, L.T. Schelhas, G. Ceder, W. Tumas, G. Teeter, and A. Zakutayev: $\mathrm{Zn}_{2} \mathrm{SbN}_{3}$ : Growth and characterization of a metastable photoactive semiconductor. Mater. Sci. Res. Horiz. 6, 1669 (2019).

31. K.N. Heinselman, S. Lany, J.D. Perkins, K.R. Talley, and A. Zakutayev: Thin film synthesis of semiconductors in the $\mathrm{Mg}_{-}$ Sb-N materials system. Chem. Mater. 31, 8717 (2019).

32. Y. Han, S. Siol, Q. Zhang, and A. Zakutayev: Optoelectronic properties of strontium and barium copper sulfides prepared by combinatorial sputtering. Chem. Mater. 29, 8239 (2017).

33. Y. Han, B.E. Matthews, D.M. Roberts, K. Talley, S. Bauers, C.L. Perkins, Q. Zhang, and A. Zakutayev: Combinatorial nitrogen gradients in sputtered thin films. ACS Comb. Sci. 20, 436 (2018).

34. A. Zakutayev, F.J. Luciano, V.P. Bollinger, A.K. Sigdel, P.F. Ndione, J.D. Perkins, J.J. Berry, P.A. Parilla, and D.S. Ginley: Development and application of an instrument for spatially resolved Seebeck coefficient measurements. Rev. Sci. Instrum. 84, 53905 (2013).

35. K.R. Talley, S.R. Bauers, C.L. Melamed, M.C. Papac, K.N. Heinselman, I. Khan, D.M. Roberts, V. Jacobson, A. Mis, and G.L. Brennecka: COMBIgor: Data analysis package for combinatorial materials science. ACS Comb. Sci. 21, 537 (2019).

36. A. Zakutayev, N. Wunder, M. Schwarting, J.D. Perkins, R. White, K. Munch, W. Tumas, and C. Phillips: An open experimental database for exploring inorganic materials. Sci. Data 5, 180053 (2018)

37. G. Kresse and J. Furthmüller: Efficient iterative schemes for $a b$ initio total-energy calculations using a plane-wave basis set. Phys. Rev. B 54, 11169 (1996).

38. G. Kresse and D. Joubert: From ultrasoft pseudopotentials to the projector augmented-wave method. Phys. Rev. B 59, 1758 (1999). 
39. J. Harl and G. Kresse: Cohesive energy curves for noble gas solids calculated by adiabatic connection fluctuation-dissipation theory. Phys. Rev. B 77, 45136 (2008).

40. J.P. Perdew, K. Burke, and M. Ernzerhof: Generalized gradient approximation made simple. Phys. Rev. Lett. 77, 3865 (1996).

41. A. Zunger, S-H. Wei, L.G. Ferreira, and J.E. Bernard: Special quasirandom structures. Phys. Rev. Lett. 65, 353 (1990).

42. M. Shishkin and G. Kresse: Implementation and performance of the frequency-dependent GW method within the PAW framework. Phys. Rev. B 74, 35101 (2006).
43. S. Lany: Band-structure calculations for the $3 \mathrm{~d}$ transition metal oxides in GW. Phys. Rev. B 87, 085112 (2013).

44. S. Lany: Semiconducting transition metal oxides. J. Phys.: Condens. Matter 27, 283203 (2015).

45. B.E. Matthews, A.M. Holder, L.T. Schelhas, S. Siol, J.W. May, M.R. Forkner, D. Vigil-Fowler, M.F. Toney, J.D. Perkins, and B.P. Gorman: Using heterostructural alloying to tune the structure and properties of the thermoelectric $\mathrm{Sn}_{1-x} \mathrm{Ca}_{x} \mathrm{Se}$. J. Mater. Chem. A 5, 16873 (2017). 